\title{
Editorial
}

\section{Special issue on image and video coding beyond standards}

\section{Why Beyond Standards?}

Today's visual information coding standards or de facto adopted methods are the fruits of a tremendous evolution, over the past decades, in several converging fields, from information theory to signal processing and computer science. Major milestones like arithmetic coding or wavelet theory have paved the way to efficient coding methods. The optimality of the so-called classical coding methods can however be questioned. Two major reasons explain the increasing importance of a daunting question: What's next?

First, the compression efficiency provided by block-based DCT or wavelets is not optimal, especially at low bit rates. The use of DCT is based on an approximate Gaussian distribution assumption, and wavelets, though optimal for monodimensional signals, loose some efficiency with multi-dimensional data such as images or video. This observation opens the door to new transform coding methods and data representation, which all emphasize the need for high adaptivity to input signal, the ability to capture true multi-dimensional features like oriented edges and an efficient data encoding.

Second, the emergence of a vast variety of visual applications in network-lossy environments highlighted the weaknesses of the classical methods in terms of error resilience and scalability. These problems are being addressed by the addition of tools to improve the standard schemes and thus provide error robustness and scalable content. However, the initial and still underlying design of these coders is not targeted. Since visual information does not obey a flat hierarchy, the optimality can only be reached by considering the end-to-end system as proposed by joint source-channel(-client) coding methods.
This special issue does not pretend to provide an exhaustive coverage of the visual coding field. Rather, the seven papers provide an overview of the new techniques which may become parts of next information coding standards.

\section{Contributions}

Though this issue mostly targets visual information coding, it was our feeling that audio coding should not be left aside. On the one hand the success of mp3 as a standardized technique prepared the pathway for new standards. On the other hand we thought it would be important to cover the media trilogy: audio, image and video. This is the reason why the first paper of this special issue focuses on audio coding. Daudet and Torresani propose a new encoding approach based upon hybrid models, without any prior signal segmentation. The method is applied to audio signals but similar strategy may apply to other signals types, like images, which also exhibit significantly different features.

The second paper by Candes and Guo describes newly invented ridgelets and curvelets transforms, which combine ideas of multi-scale analysis and geometry. It addresses the problem of efficiently representing images as truly bi-dimensional objects. The paper presents an effective numerical implementation, as well as a remarkable potential in image compression, restoration and de-noising. A minimum Total Variation Synthesis is proposed as an efficient Edge-Preserving image reconstruction scheme.

The third paper by Cho and Pearlman introduces an error resilient video streaming format based on a three-dimensional SPIHT encoder. It proposes a new method of partitioning wavelets coefficients in order to achieve error resilience and ease the concealment, 
while maintaining an embedded bitstream. An unequal error protection scheme then provides a improved efficiency against bit errors.

Tanaka and Yamashita then present the theory and design of an orientation adaptive lapped biorthogonal transform for image coding. This new coding method can advantageously replace the commonly-used block-based DCT since it significantly reduces blocking artifacts while improving visual quality around edges and lines.

In the following paper, Carvalho, da Silva and Finamore propose a new multi-dimensional signal compression method based on multi-scale recurrent patterns. The multi-dimensional signal is recursively segmented into variable-length vectors, further encoded using expansions and contractions of vectors in an adaptive dictionary. This very effective adaptation method allows for improved performance compared to state-of-the-art image coders.

The sixth paper by Aiazzi, Alparone and Baronti targets (near-)lossless compression of monochrome images. It proposes a DPCM method based on a classified linear-regression prediction followed by context-based arithmetic coding of the residuals. This new scheme provides impressive performances on medical images and compares advantageously to literature advanced methods and current standards.

In the last paper, Santa-Cruz and Ebrahimi propose a new method for compressing NURBS 3D models with a small and controllable loss. The scheme is based on a DPCM coder with different predictors for knots and control points, followed by uniform scalar quantization and bitplane arithmetic coder. The new scheme shows significantly better compression performance and visual quality than standard methods.

Pierre Vandergheynst Swiss Federal Institute of Technology Lausanne, Switzerland E-mail address: pierre.vandergheynst@epfl.ch

Pascal Frossard IBM TJ Watson Research Center Yorktown Heights, USA E-mail address: frossard@us.ibm.com 